\section{L'état actuel des vieilles sapinières à Hylocomium à la forêt Montmorency de l'Université Laval}

\author{
Par GILBERT PAILLE
}

\section{Résumé}

Des sapinières parvenues à maturité ont été décrites et mesurées au moyen de 53 places d'étude d'un cinquième d'acre dans la Forêt Montmorency de l'Université Laval. Cette étude avait pour but de comparer l'état actuel des peupplements à l'état normal. Les comparaisons avec les tables de production normale ont porté sur (1) le nombre et la distribution des tiges par classes de diamètre, (2) la densité absolue et la densité relative des peuplements, (3) le volume brut et l'accroissement annuel moyen.

On a trouvé que les sapinières situées sur des stations de deuxième qualité (1) comportent un grand nombre de tiges de faible diamètre, (2) ont une densité qui permet l'établissement sous couvert d'une régénération naturelle abondante, (3) s'accroissent à un rythme très inférieur au potentiel des stations.

Pour imiter l'oeuvre de la nature, il faudrait appliquer à ces peuplements équiennes un système de coupes progressives. La période de révolution devrait être inférieure à 70 ans, de façon à éviter les pertes par carie et mortalité naturelle.

\section{Abstract}

The present status of old balsam fir stands (Hylocomium-Oxalis site-type) has been studied on sites of second quality (index of 40 feet at 50 years) by means of 1/5-acre random sample plots. Comparisons with normal yield tables indicate that, between the age of 70 and 90 years, these stands show 1) too many stems per acre, mainly

\footnotetext{
${ }^{1}$ Etude basée sur une partie des mesures faites par l'auteur, pour la préparation d'une thèse de maîtrise ès sciences, sous la direction du Dr Paul-Emile Vézina, professeur à la Faculté de Foresterie et de Géodésie de l'Université Laval.
}

Candidat ou doctorat, Faculté de Foresterie, Université de Colombie-Britannique, Vancouver.

ODC 228.5: (7 14)

Manuscript received March 1968.

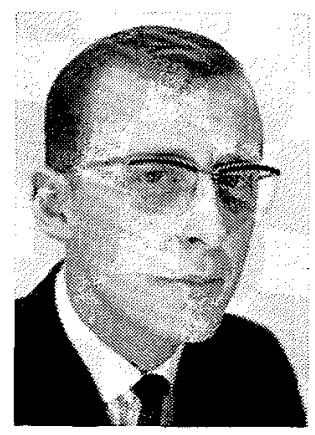

due to abundant ingrowth, 2) a constant average stand d.b.h., 3) a loss of 30 sq.ft. per acre of basal area, and 4) a reduction of the mean annual increment in merchantable volume from 33 to 22 cu.ft. per acre.

It is concluded that these site class II balsam fir stands are already mature, rapidly deteriorating, and failing to occupy the station fully. Consequently, they should be managed on rotations shorter than 70 years. Thinnings might be conducted 15 years before the final cutting to salvage otherwise heavy losses by natural mortality.

\section{Introduction}

A l'aide des tables de production de Vézina et Linteau (1968), il est possible de comparer l'état actuel des sapinières à Hylocomium à leur état normal. Cette comparaison permet de formuler certaines prescriptions sylvicoles pour maintenir les stations à un haut niveau de productivité et obtenir une production maximum en matière ligneuse récupérable.

\section{Milieu}

Les peuplements de sapin baumier (Abies balsamea (L.) Mill.) étudiés croissent sur des stations de deuxième qualité (indice de fertilité variant de 36 à 45 pieds à 50 ans) dans la Forêt Montmorency de I'Université Laval, à 50 milles au nord de Québec.

Ils ont pour origine des châblis intensifs. Ils sont composés en majeure partie de sapin rendu à maturité, de bouleau à papier (Betula papyrifera Marsh.) de faible qualité et d'épinette blanche (Picea glauca (Moench) Voss). Ils forment des peuplements équiennes où la carie semble jouer un rôle important, si l'on en juge par les défauts externes 
des arbres (Lortie 1968). Ils font partie de l'association du sapin à l'Oxalis, décrite par Lafond (1967). La strate muscinale est composée surtout d'Hylocomium proliferum et la strate herbacée d'Oxalis montana.

Ils se situent à des altitudes variant de 1,800 à 3,200 pieds, sur des pentes inférieures à 50 pour 100. La précipitation annuelle dans cette région est de 52 pouces, dont 170 pouces de neige. La saison sans gel est de 60 jours environ. Les sols sont des podzols humiques orthiques (Swain) bien drainés, formés sur dépôts glaciaires, et des podzols humiques à gley (Colligan) mal drainés, sur dépôts fluvio-glaciaires. La litière est composée d'un mor granuleux assez mince et acide.

\section{Etude}

Cinquante-trois places d'étude rectangulaires d'un cinquième d'acre ont été localisées au hasard dans ces sapinières à Hylocomium. Dans chaque place, les mesures suivantes ont été effectuées: (1) diamètre des tiges par essence à hauteur de poitrine; (2) hauteur totale de 5 arbres dominants ou co-dominants; (3) âge à la souche des mêmes sujets; (4) pente du terrain. A partir de ces mesures, on a calculé (1) le nombre de tige à l'acre, (2) la surface terrière, en pieds carrés à l'acre, (3) le diamètre moyen, (4) le volume et (5) l'accroissement. Le tarif de cubage utilisé a été construit pour des peuplements semblables du Parc des Laurentides.

\section{Résultats}

1. Nombre de tiges et diamètre.

La figure 1 montre que les sapinières à Hylocomium renferment plus de tiges à l'acre que les sapinières de densité normale de même type et de méme âge. Ce surplus est attribuable à la trop grande abondance d'arbres dont le diamètre est inférieur à 3 pouces. Par contre, dans les classes supérieures de diamètre, elles présentent un déficit en nombre d'arbres à l'acre par rapport à la normale. Ce phénomène est illustré à la figure 2 .

L'établissement massif de jeunes tiges sous le peuplement mûr et le manque de sujets de fort diamètre ont pour effet de maintenir, entre 70 et 90 ans, le diamètre moyen du peuplement à un niveau stable et très inférieur au niveau normal. Ce processus semble même s'accentuer avec l'âge, comme indiqué à la figure 3 .

\section{Densité}

Les courbes de la figure 4 montrent que la densité des sapinières à Hylocomium diminue fortement avec l'âge. En effet, passé l'âge de 70 ans, elles perdent naturellement et rapidement 30 pieds carrés de surface terrière et leur densité relative passe de 99 à 82 pour 100 en 20 ans.

\section{Volume et accroissement}

Le volume des sapinières étudiées est inférieur au volume normal par une marge qui varie de 1,200 à 1,600 pieds cubes, tel qu'indiqué à la figure 5 . De plus, le volume de bois résineux ne s'accroit en moyenne que de 22 à 33 pieds cubes par année (figure 6).

\section{Discussion}

L'observation de ces résultats suggère que les sapinières à Hylocomium atteignent leur maturité absolue avant l'âge de 70 ans. En effet, passé cet âge, l'accroissement annuel moyen diminue fortement de même que la densité. De plus, à cet âge, une régénération naturelle abondante est déjà établie sous le couvert du peuplement principal et constituée de 400 à 500 tiges de moins de 3 pouces de diamètre. Ces signes indiquent que le peuplement se dégrade rapidement après 70 ans.

Les résultats permettent aussi d'estimer à plus de 100 pieds cubes à l'acre la quantité de matière ligneuse qui est perdue par ralentissement de l'accroissement et mortalité naturelle, entre l'âge de 70 et 90 ans. L'accroissement est ralenti parce que le peuplement principal occupe avec de moins en moins d'efficacité la station à mesure qu'il vieillit. L'apparition hâtive (Smerlis 1961) et le développement rapide de la carie, qui attaque 75 pour 100 des tiges d'âge avancé (Giguère 1964) et les rend très vulnérables au vent, semble être la principale cause de mortalité naturelle.

TABLEAU 1 VALEURS MINIMUM, MAXIMUM, MOYENNE ET ERREUR STANDARD DES PRINCIPALES CARACTERISTIOUES DES VIEILLES SAPINIERES EN FONCTION DE LEUR ÂGE

\begin{tabular}{|c|c|c|c|}
\hline \multirow{2}{*}{$\begin{array}{l}\text { Caractéristiques } \\
\text { des peuplements }\end{array}$} & \multicolumn{3}{|c|}{ Âge des peuplements } \\
\hline & 70 & (annees) & 90 \\
\hline \multicolumn{4}{|l|}{ Nombre de tiges } \\
\hline min. & 460 & 365 & 600 \\
\hline $\max$ & 2160 & 1830 & 1570 \\
\hline moy. & 1235.8 & 1035.5 & 1012.9 \\
\hline erreur st. & 507.1 & 322.4 & 341.8 \\
\hline \multicolumn{4}{|l|}{$\begin{array}{l}\text { Diamètre moyen } \\
\text { (pouces) }\end{array}$} \\
\hline min. & 3.8 & 3.7 & 3.1 \\
\hline $\max$ & 7.8 & 6.8 & 6.7 \\
\hline moy. & 5.3 & 5.3 & 5.3 \\
\hline erreur st. & 1.1 & 0.8 & 1.2 \\
\hline \multicolumn{4}{|l|}{$\begin{array}{l}\text { Surface terrière } \\
\text { (pi carrés /acre) }\end{array}$} \\
\hline min. & 131.4 & 86.8 & 83.4 \\
\hline $\max$ & 230.6 & 205.3 & 176.4 \\
\hline moy. & 167.6 & 152.7 & 142.2 \\
\hline erreur st. & 23.1 & 28.2 & 32.0 \\
\hline \multicolumn{4}{|c|}{$\begin{array}{l}\text { Volume marchand } \\
\text { résineux } \\
\text { (pi cubes/acre) }\end{array}$} \\
\hline min. & 1449 & 898 & 978 \\
\hline $\max$ & 3207 & 2889 & 2619 \\
\hline moy. & 2377.9 & 2016.4 & 2023.0 \\
\hline erreur st. & 429.6 & 549.1 & 682.9 \\
\hline \multirow{2}{*}{\multicolumn{4}{|c|}{$\begin{array}{l}\text { Accroissement anuel } \\
\text { moyen résineux } \\
\text { (pi cubes/acre) }\end{array}$}} \\
\hline & & & \\
\hline min. & 22.6 & 11.0 & 11.1 \\
\hline $\max$ & 44.5 & 36.5 & 28.8 \\
\hline moy. & 33.4 & 26.1 & 22.6 \\
\hline erreur st. & 5.0 & 6.8 & 7.7 \\
\hline
\end{tabular}

February 1969 The Forestry Chronicle 
SAPINIERE
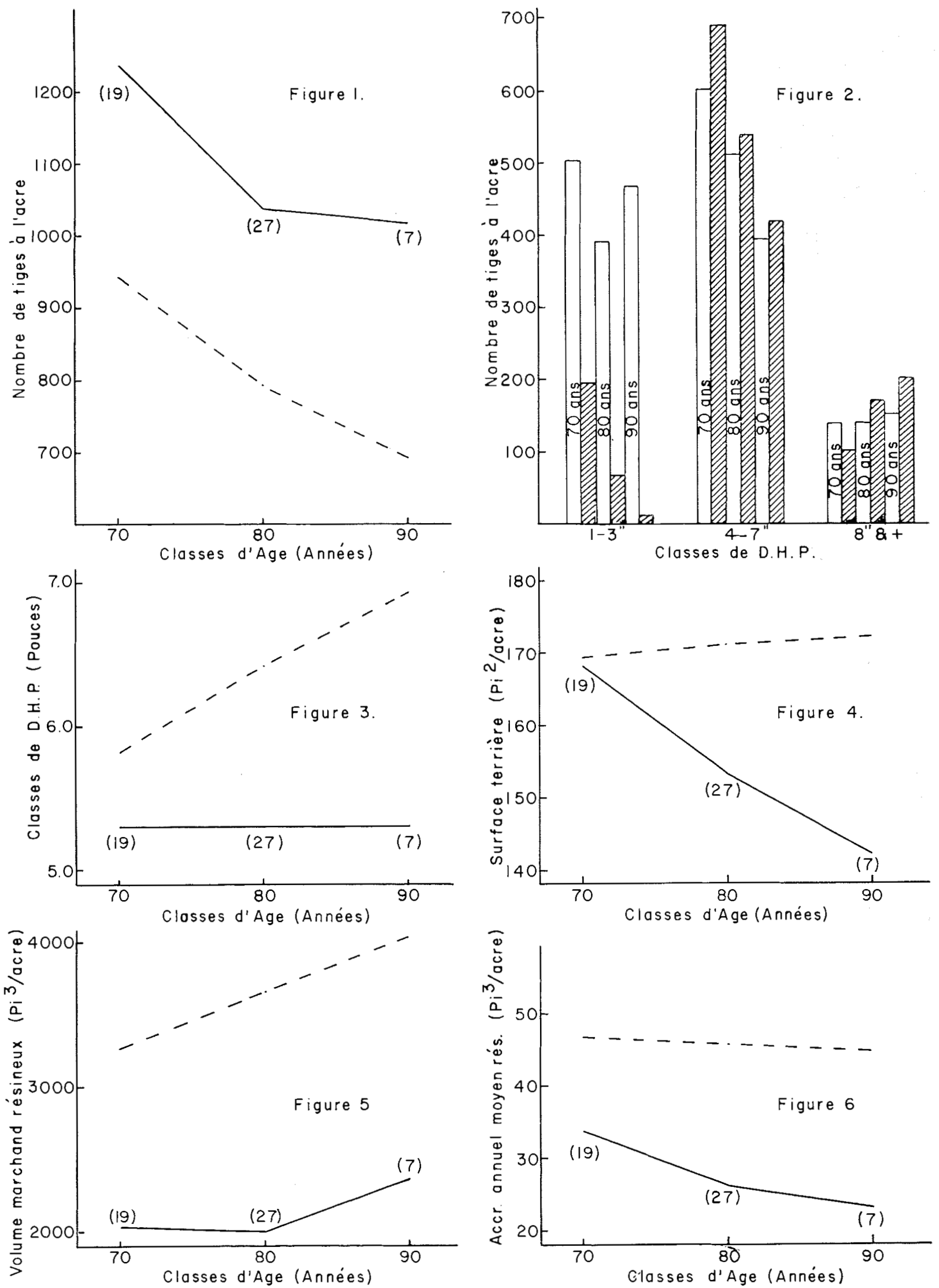

$\square-$ Peuplement étudié

D - - Peuplement normal

( ) Nombre de places d'étude 
Les résultats présentés indiquent enfin la nature des traitements culturaux qu'il faudrait appliquer aux sapinières pour les maintenir dans un état qui soit plus près de la normale, du double point de vue qualitatif et quantitatif. Tel que le suggèrent les études de Paillé (1968) sur l'aménagement intensif des peuplements de sapin, un système de coupes progressives devrait être appliqué. Des éclaircies fortes par le haut, effectuées au moins 15 ans avant la coupe finale auraient pour principal effet de permettre la récupération des tiges qui sont appelées à disparaître naturellement. Cette période d'application des traitements est suggérée par l'âge de la régénération naturelle établie dans les peuplements non aménagés. Elle pourrait aussi être déterminée en suivant le régime d'éclaircies proposé par Vézina (1964).

Le degré d'intensité de l'aménagement à pratiquer pour obtenir des résultats tangibles n'aurait pas besoin d'être très considérable puisque, même sans aménagement, un certain nombre de peuplements présentent des structures qui se rapprochent de la normale (tableau 1).

\section{Conclusion}

A la lumière de cette étude, on constate que passé l'âge de 70 ans, les peuplements de sapin à $\mathbf{H y -}$ locomium perdent rapidement le contrôle de la sta. tion. Par contre, l'ouverture dans le couvert principal permet l'établissement d'une régénération naturelle dense.

Ainsi, deux conclusions principales peuvent être retenues:

\section{Références}

GIGUERE, M. 1964. Etude de la carie et des défauts externes des arbres dans un peuplement de sapin (Dry-Ox 80 ans). Thèse de maitrise, Fac. d'Arp. et de Gén. For., Univ. Laval, Québec. $127 \mathrm{pp}$.

LAFOND, A. 1967. Notes pour l'identification des types forestiers des concessions de la Quebec North Shore Paper Co. 3 e éd., Baie-Comeau. 93 pp.

LORTIE, M. 1968. Quelques défauts indicateurs de carie chez le sapin baumier. Fonds de Rech. For., Univ. Laval, Québec. Bull. No. 11. 38 pp.
1. La maturité absolue est atteinte avant l'âge de 70 ans dans les sapinières à Hylocomium.

2. L'application d'un système de coupes progressives aurait pour effet d'augmenter sensiblement la quantité de bois récupérable par unité de surface. Ce traitement contribuerait aussi à la production de billes de plus grande dimension et de meilleure qualité.

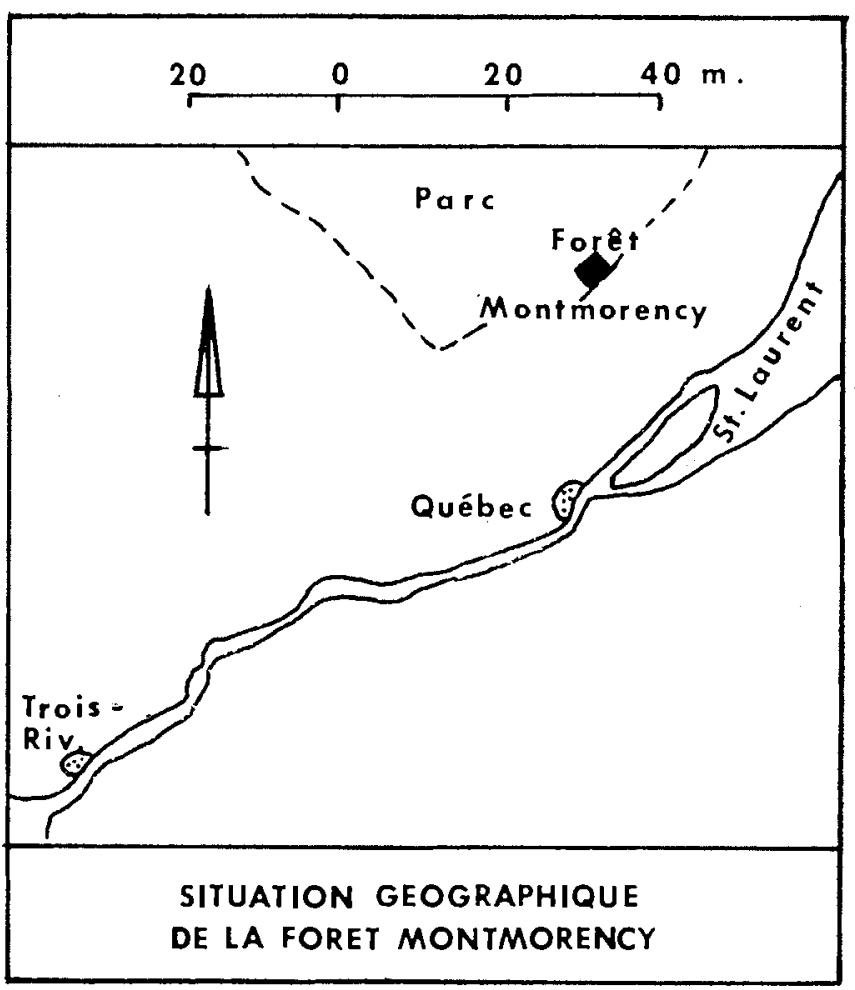

PAILLE, G. 1968. Plan d'aménagement intensif des peuplements mûrs et surannés à la Forêt Montmorency. Thèse de maitrise, Fac. For. et Géo., Univ. Laval, Québec. 103 pp.

VEZINA P. E. 1964. Derivation of thinning regimes througi use of a model for balsam fir in Quebec. For. Chron. 40 (3): $362-71$.

VEZINA, P. E. and A. LINTEAU. 1968. Growth and yield of balsam fir and black spruce in Quebec. Canada Dept. For. and Rur. Dev., For. Br., Inf. Rep. Q-X-2. $58 \mathrm{pp}$. 